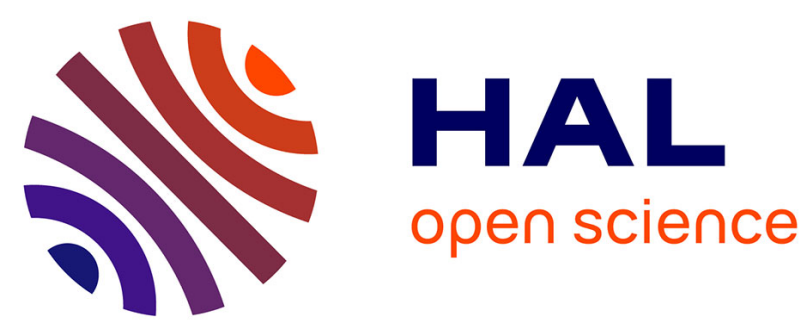

\title{
A Wearable Machine Learning Solution for Internet Traffic Classification in Satellite Communications
}

Fannia Pacheco, Ernesto Expósito, Mathieu Gineste

\section{To cite this version:}

Fannia Pacheco, Ernesto Expósito, Mathieu Gineste. A Wearable Machine Learning Solution for Internet Traffic Classification in Satellite Communications. ICSOC 2019: The 17th International Conference on Service-Oriented Computing, Oct 2019, Toulouse, France. pp.202-215, 10.1007/978-3030-33702-5_15. hal-02423379

\section{HAL Id: hal-02423379 https://hal.science/hal-02423379}

Submitted on 26 Jan 2020

HAL is a multi-disciplinary open access archive for the deposit and dissemination of scientific research documents, whether they are published or not. The documents may come from teaching and research institutions in France or abroad, or from public or private research centers.
L'archive ouverte pluridisciplinaire HAL, est destinée au dépôt et à la diffusion de documents scientifiques de niveau recherche, publiés ou non, émanant des établissements d'enseignement et de recherche français ou étrangers, des laboratoires publics ou privés. 


\title{
A wearable Machine Learning solution for Internet traffic classification in Satellite Communications
}

\author{
Fannia Pacheco ${ }^{1}$, Ernesto Exposito ${ }^{1}$, and Mathieu Gineste ${ }^{2}$ \\ 1 Univ Pau \& Pays Adour, E2S UPPA, LIUPPA, EA3000, Anglet, 64600, France \\ $\{f \cdot$ pacheco, ernesto.exposito-garcia\}@univ-pau.fr \\ 2 Département : Business Line Telecommunication, R\&D départment, Thales Alenia \\ Space, TOULOUSE, 31100, France. \\ mathieu.gineste@thalesaleniaspace.com
}

\begin{abstract}
In this paper, we present an architectural framework to perform Internet traffic classification in Satellite Communications for QoS management. Such a framework is based on Machine Learning techniques. We propose the elements that the framework should include, as well as an implementation proposal. We define and validate some of its elements by evaluating an Internet dataset generated on an emulated Satellite Architecture. We also outline some discussions and future works that should be addressed to have an accurate Internet classification system.
\end{abstract}

Keywords: Internet traffic classification - Machine Learning - Satellite Communications · Deep packet inspection.

\section{Introduction}

Internet traffic classification is a group of strategies that aims at classifying the Internet traffic into predefined categories, such as normal or abnormal traffic, the type of application (streaming, web browsing, VoIP, etc.) or the name of the application (YouTube, Netflix, Facebook, etc.). Network traffic classification is important in Satellite communication principally to manage bandwidth resources and to ensure Quality of Service (QoS) requirements.

Traffic classification is widely implemented by Deep Parquet Inspection(DPI) solutions. Most of the commercial solutions use this technology for traffic management. DPI performs matching between the packet payload and a set of stored signatures to classify network traffic. However, DPI fails when privacy policies and laws prevent accessing the packet content, as well as the case of protocol obfuscation or encapsulation. To overcome the previous issues, Machine Learning (ML) emerged as a suitable solution, not only for the traffic classification task but also for prediction and new knowledge discovery. In this context, statistical features of IP flows are commonly extracted and stored from network traces to generate historical data. In this way, different ML models can be trained with this historical data, and new incoming flows can be analyzed with such models. 
In satellite networks, Internet traffic management is a key task due to it allows improving the QoS. Commonly, traffic data is captured from satellite Internet Service Providers (ISPs). The works in this area aim to classify and to analyze Internet traffic in large networks $[6,14,12]$. The principle is to deploy passive monitoring points to perform traffic classification. These monitoring points can be at routers [6] or points of presence (PoPs) [12] of large ISP networks. Another emerging approach is the use of Software-defined networks(SDNs) in satelliteterrestrial networks. In SDNs, traffic classification can be easily deployed in the SDN' master controllers as it is exposed in $[8,1]$.

The authors outlined the complete process to achieve Internet traffic classification in the survey paper [10]. Therefore, this approach focuses its attention on developing a framework that can be deployed in a Satellite architecture. Such a framework comprises all the necessary elements to achieve the goal, as well as additional components that should be integrated to assure a robust classification tool. We propose a hierarchical classification system based on ML, which treats encryption and flow patterns differently. We deploy the solution in a low-level language that allows having an efficient and fast classification output. We also compare our approach with a well-known DPI solution called nDPI [2]. Finally, we set discussions about some important components that are in development; for instance, the treatment of tunneled connections and the evolution of the Internet network.

\section{QoS management in Satellite Communications}

At this point, we start by introducing the general reference model to provide Satellite Communications. This model will serve us as guidance to find the requirements to integrate ML in such architecture. A typical reference model of a multi-gateway Satellite architecture is shown in Figure 1 [3]. This model is divided into two main blocks: Satellite access network and Satellite core network. On the one hand, in the Satellite access network, a variety of network topologies can be used to the connectivity of the elements; these included the Satellite gateways and terminals. On the other hand, in the Satellite core network, an aggregate network allows interconnecting with other operators, corporations, and Internet Service Providers (ISPs) through Points of Presence (PoPs).

Two main components of such model are described below:

- Satellite Terminal (ST): its function is to deliver broadband access to enduser equipment through IP routers and/or Ethernet switches.

- Satellite Gateway (GW): this component is in charge of deploying user plane functions such as packet routing and forwarding, interconnection to the data network, policy enforcement, and data buffering. These functionalities are coordinated by the control and management systems of the Satellite network. The GW is composed of forwarding and returning link (FL and RL) subsystems, and a set of network functions. These network functions include the Performance Enhancing Proxy (PEP), switching and routing interfaces for the interconnection with the Satellite core network. 


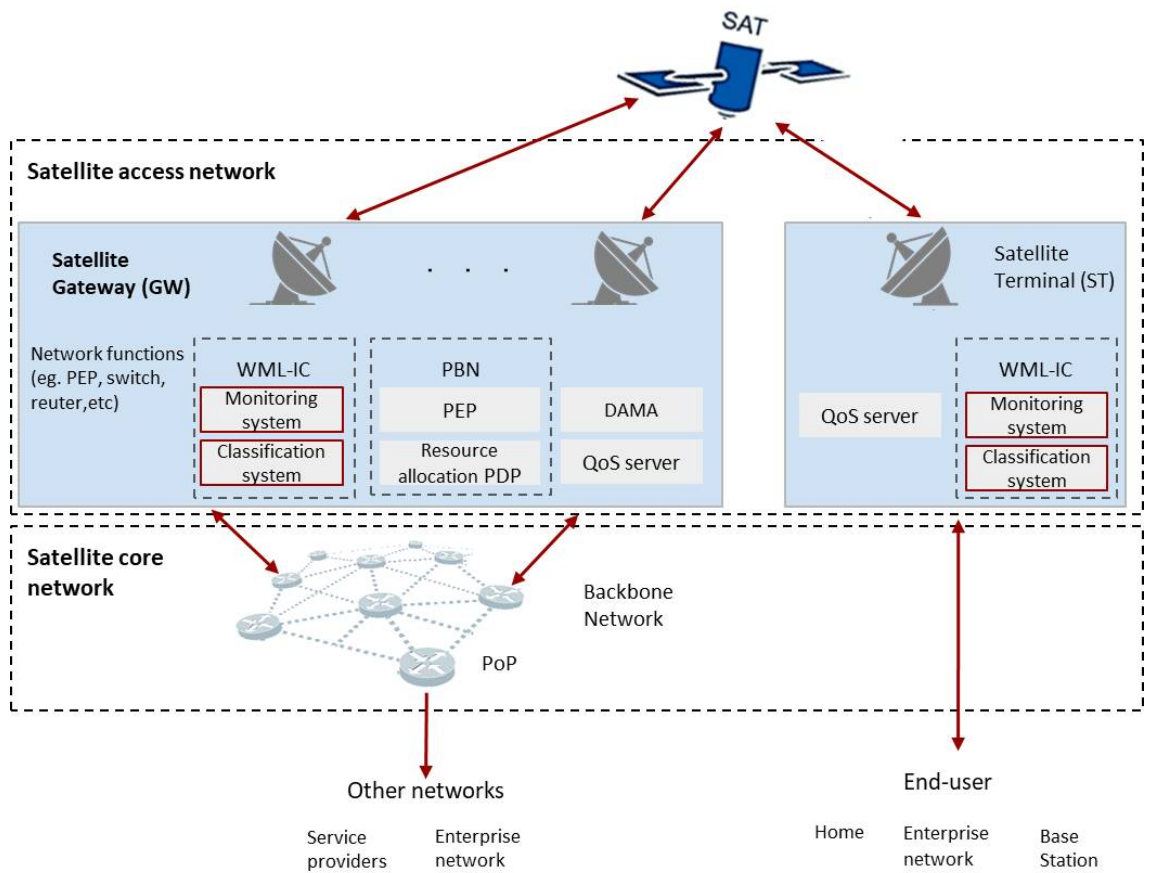

Fig. 1: Reference model of a multi-gateway Satellite network architecture.

One of the main objectives of this architecture is to provide a reliable communication system between different entities. However, improving the Quality of Service (QoS) and Quality of Experience (QoE) of their users is of paramount importance for network administrators. In principle, these last objectives can be achieved by manipulating the network functions. More specifically, a Policy Based Network (PBN) Architecture is deployed at this stage to perform traffic management [7]. To improve the QoS, one of the most common and accepted actions is to fulfill a set of requirements that can be executed by profiling Internet traffic $[5,13]$. This idea parts from the assumption that some Internet traffic is more sensitive to information loss and delay, such as Internet calling or video conference. In contrast, Internet browsing or file downloads are less pruned to be affected by these error conditions.

Following this idea, the main goal of our proposal is to correctly profile the Internet communications, to later transmit this information to a PBN that will take the necessary actions for QoS management. Hence, in Figure 2, we add two new elements to allow Internet traffic classification: Monitoring and Classification system. The resulting classification is forwarded to the PBN. In the figure above, we also show three essential components in the PBN: PDP, Resource allocation PEP, and QoS server. Briefly speaking, traffic classification is signaled to the PDP that will define what QoS policy should be applied to 
a flow or set of flows. The QoS policy is then sent to the Policy Enforcement Functions (PEFs) such as the QoS servers and Resource allocation PEP. The QoS server applies the QoS rules on the equipment that handles the traffic (GW, ST), and the Resource allocation PEP provides the resource when needed to the User Terminal. To instantiate the resource allocation toward the terminal, the Resource allocation PEP is composed of other functional elements that are omitted due to they do not affect the modeling of our approach. In particular, what will be of paramount importance is correctly handling the traffic monitored and signaling the classification to the PBN.

\section{Architecture design}

Making an abstraction of the elements in a real Satellite network distribution, the primary steps to achieve Internet traffic classification in a Satellite Architecture are:

1. Intercept Internet traffic in the GW and ST through the Passive monitoring points.

2. Perform feature extraction over the Internet flows.

3. Send the extracted features to the Classification System and mark the flows with their QoS classes.

4. Forward the classification to the PDP that will take decisions in order to improve the QoS. Then the Resource allocation PEP and the QoS servers will execute those decisions.

In order to formally define the requirements of the system, we follow the Model-Based System Engineering methodology proposed by ARCADIA and the open-source methodology tool named Capella ${ }^{3}$. Capella follows ARCADIA principles to provide methodological guidance, intuitive model editing, and viewing capabilities for Systems, Software and Hardware Architects. In Capella, the Operational analysis and System analysis help finding and defining the requirements of the system. Whereas, the Logical and Physical architectures aim at developing the solution. Figure 2 shows a System Analysis viewpoint, focused on the GW actor, developing the requirement: Provide Internet traffic classification in Satellite Communications for QoS management. We will discuss as follows the functions associated to this system analysis.

\footnotetext{
${ }^{3}$ https://www.polarsys.org/capella/
} 


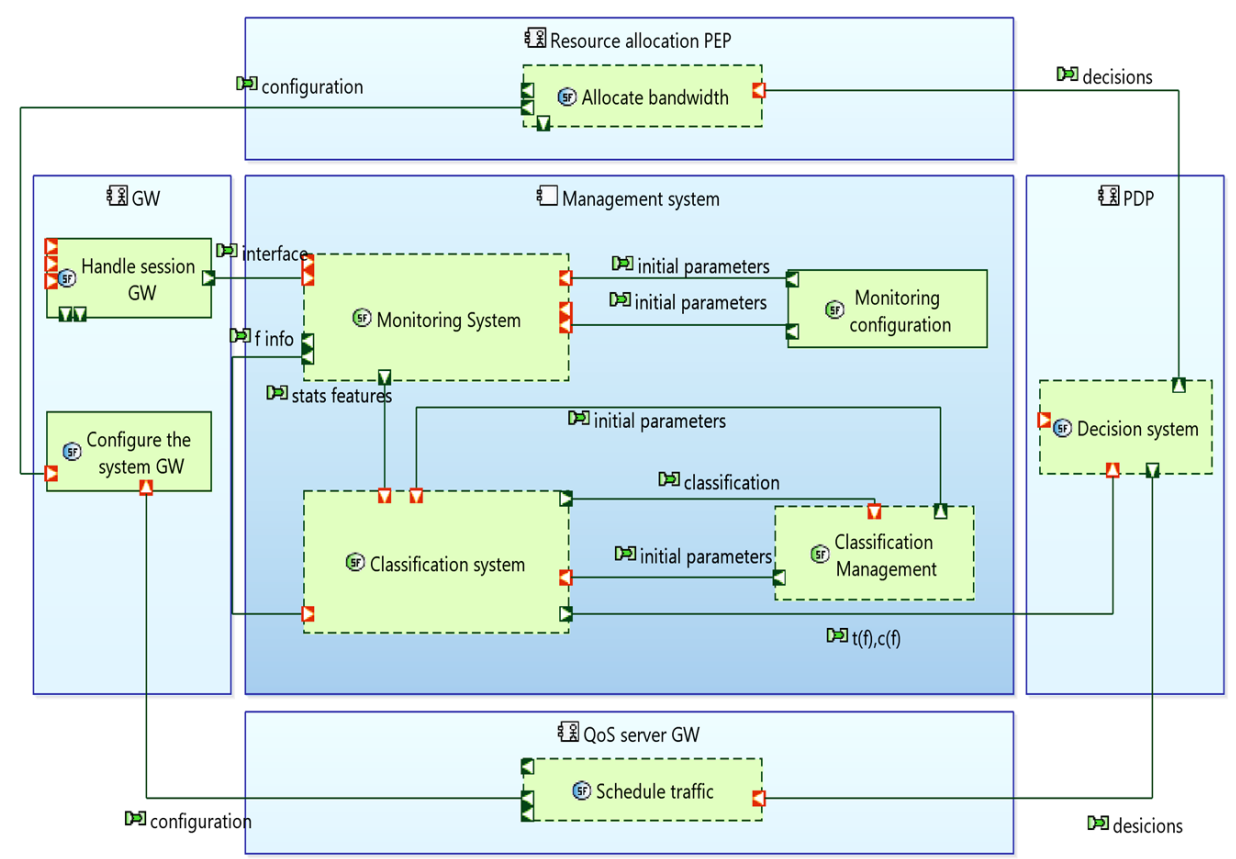

Fig. 2: System Analysis in the GW.

\subsection{Classification system}

Particularly, this system proposes an automatic and logic process to analyze traffic hierarchically. The classification system is displayed in Figure 3. Briefly speaking, the process starts performing the Offline configuration process to initialize the whole classification system (training process). In an online manner, the flow features pass through a Flow discriminator 1 (D1) that will be in charge of disjointing the non-encrypted/Encrypted flows from the tunneled flows. This separation will allow us to treat each technology differently. For instance, for the non-encrypted/Encrypted streams, classical ML models or DPI solutions (denoted as Cl1) can label the flows. Whereas, the tunneled flows will pass through another Flow discriminator 2 (D2) that separates the unitary (only one application within the tunnel) and the multiple (several applications at the same time in the tunnel). Finally, once the classifiers are actively working the Online configuration component is receiving information that can induce to change or to add models in the Model repository.

\subsection{Monitoring system}

Internet packets are captured to be organized into flows $F$. The construction of the flow is given in Figure 4. In principle, all the flows are built matching the 
F. Pacheco et al.

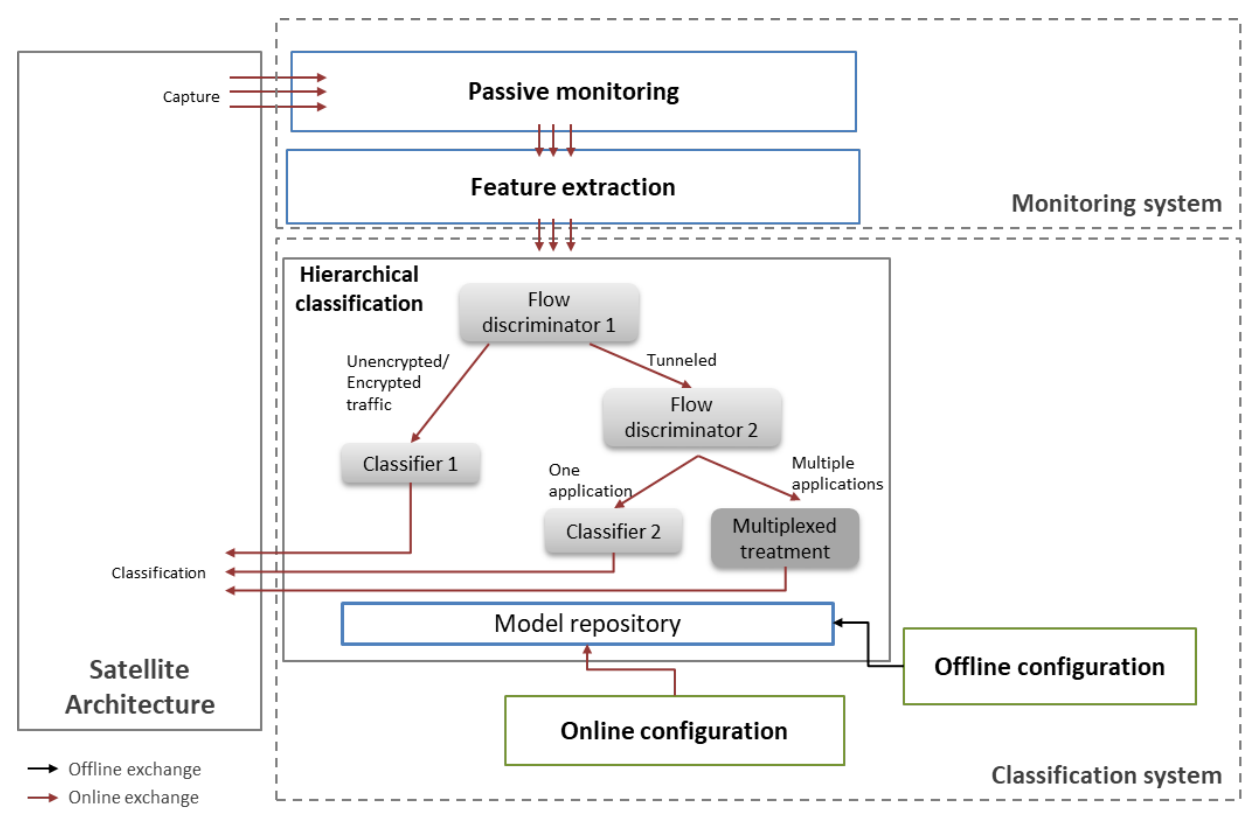

Fig. 3: Classification framework

packet's headers, source (src) and destination (dst) IPs and ports. However, when $D 1$ detected a multiplexed connection, the flow is broken into chunks of flows within a time interval, as seen in Figure 4 . Then, statistical-based features are computed for each flow to describe the communications. In brief, the properties computed are listed in Table 1. The authors studied the passive monitoring and feature extraction processes in $[11,9]$. The categorization of the packets (A, B, C, $\mathrm{D}, \mathrm{E}$, and $\mathrm{F}$ ) in Table 1 is obtained by studying the packet length distributions per class in the dataset.
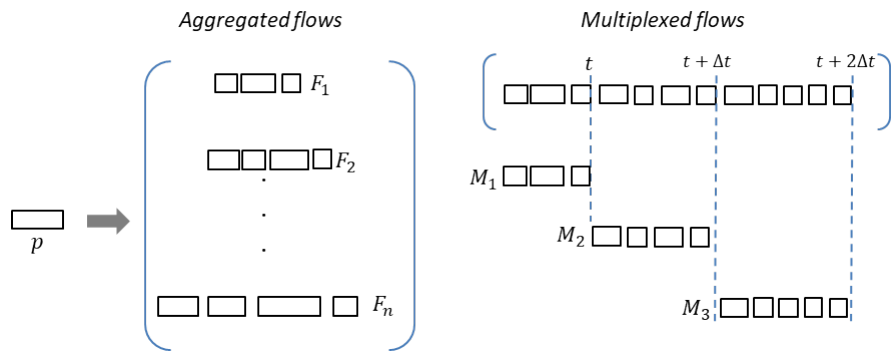

Fig. 4: Flow reconstruction. 


\begin{tabular}{|c|c|c|c|c|}
\hline Feature & Metric & Additional Information & Flow direction & Total \\
\hline pktlen-[m] & {$[\mathrm{m}]$ of the packet lengths } & $\begin{array}{l}\text { "m" refers to the metric } \\
\text { Mean, Std, Min and Max }\end{array}$ & $F, F_{s r c}$ and $F_{d s t}$ & 12 \\
\hline iat_[m] & [m] of the inter-arrival time(iat) & - & $F, F_{s r c}$ and $F_{d s t}$ & 12 \\
\hline pktlen_[cat $]_{-}[\mathrm{m}]$ & $\begin{array}{l}{[\mathrm{m}] \text { of the packet lengths per }} \\
\text { [cat] }\end{array}$ & $\begin{array}{l}\text { "cat" refers to the type } \\
\text { of packet }{ }^{a}\end{array}$ & $F, F_{s r c}$ and $F_{d s t}$ & 72 \\
\hline iat_[cat]_[m] & [m] of the iat per [cat] & & $F, F_{s r c}$ and $F_{d s t}$ & 72 \\
\hline bytes_[ $[\Delta t]$ & bytes per $[\Delta t]$ & $\begin{array}{l}\text { " } \Delta t \text { " is the time win- } \\
\text { dows }\end{array}$ & $F, F_{s r c}$ and $F_{d s t}$ & 3 \\
\hline$p k t_{-}[\Delta t]$ & packets counts per $[\Delta t]$ & - & $F, F_{s r c}$ and $F_{d s t}$ & 3 \\
\hline \multicolumn{4}{|c|}{ Total } & 174 \\
\hline
\end{tabular}

Table 1: Result of the feature extraction process

\subsection{Classification Management}

This component implements the offline and online reconfiguration. Regarding the Online reconfiguration component, this element will be in charge of evaluating the predictions performed by the classifiers. This is deployed to cope with the evolution of the network. Therefore, in an online manner, this component will evaluate if the traffic observed belongs to an existing QoS class; if so the classifier will "evolve" to offer more accurate predictions. This approach can be translated to a retraining process when new data is generated. Nonetheless, there are other approaches based on clustering that could detect class evolution.

As a final note, the current investigation does not treat the Online configuration and Multiplexed treatment due to they involve more complex tasks that will be presented in future works.

\section{Implementation design}

The implementation proposal is presented in Figure 5, with the operational and physical architecture in the same viewpoint. The subsystems proposed in Figure 5 will define how the components of the QoS management system work. For instance, the Offline configuration will be developed by the Training process and Historical data manager components, the Online configuration by the Model manager and the Incremental Learning Model(ILM) manager components. In addition to this, we define two new physical components that will be necessary for the implementation: A $G W$ server that will be in charge of taking the Internet traffic for its further classification, and a Management Server that will handle offline and online configurations.

It is worth mentioning that the functions of the $G W$ server and the Management Server can be comprised in the GW entity. This is modifiable according to the resources available in the real Satellite Architecture. On the other hand, all the functions concerning the Classification system are comprised in Framework: 
which in turn is a library developed for this aim. For what concerns the sniffer, we use existing solutions such as Libcap ${ }^{4}$ for performing the sniffing. Then, we add the Flow reconstruction and Feature Extraction behaviors. The ML models $D 1, C l 1, D 2$ and $C l 2$ will be selected in the experimental section.

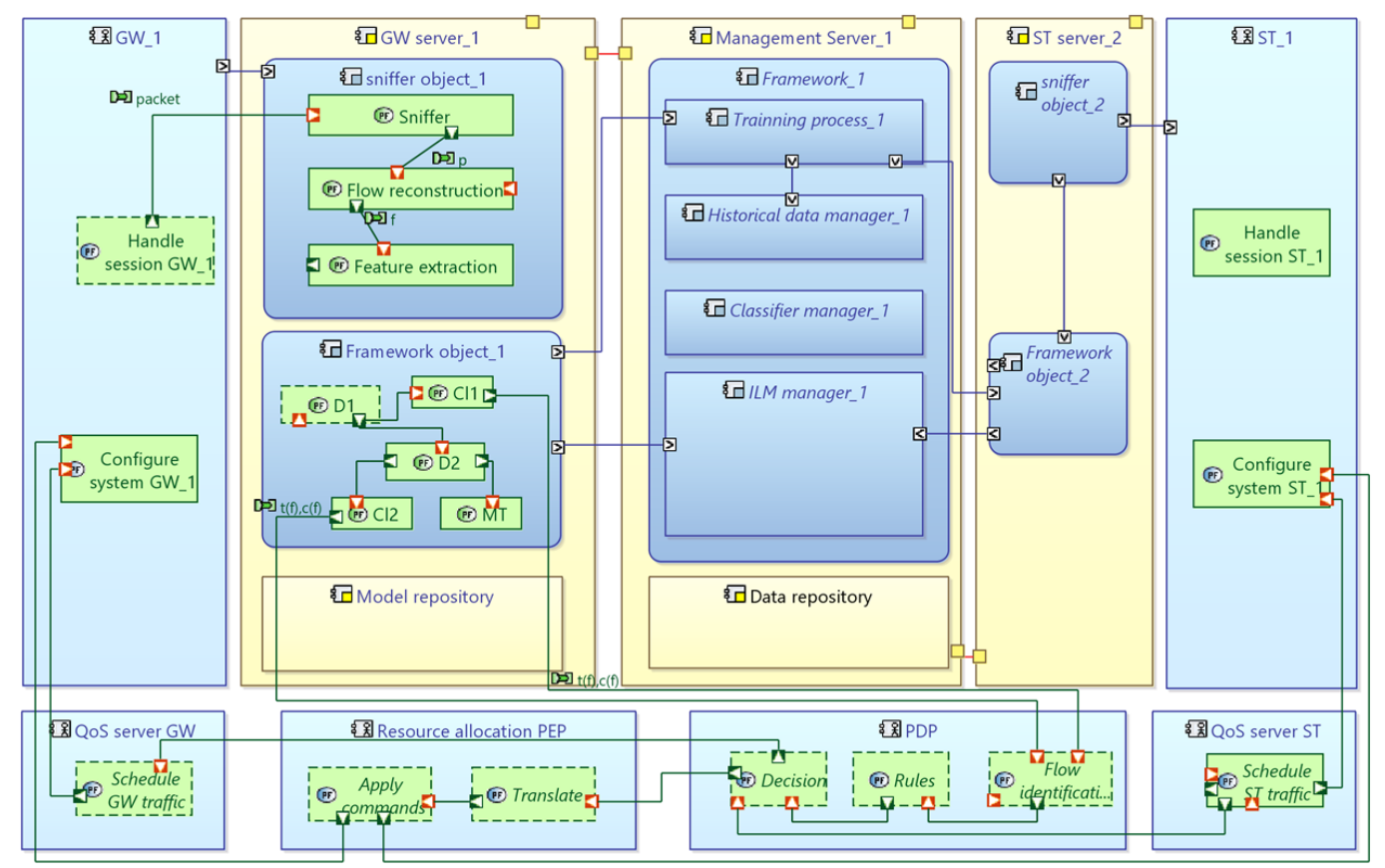

Fig. 5: System Analysis in the GW.

As an additional comment, the reader can notice that the proposed implementation can be easily replicated in the ST component, as well as in different network components where packet monitoring is feasible.

\footnotetext{
$\overline{4}$ https://www.tcpdump.org/
} 


\section{Emulated Satellite Internet Traffic}

This data set is a private dataset called SAT data. The model of a multi-gateway Satellite network in Figure 6 with one ST and one GW was set over OpenSAND ${ }^{5}$, which is a platform to emulate Satellite Communications. In addition to this, a VPN configuration is disposed between the ST and the GW, to emulate tunneled communications. Several applications were launched and captured by OpenBACH ${ }^{6}$. The user behavior was mimicked by using Selenium ${ }^{7}$, which is a tool to test web applications.

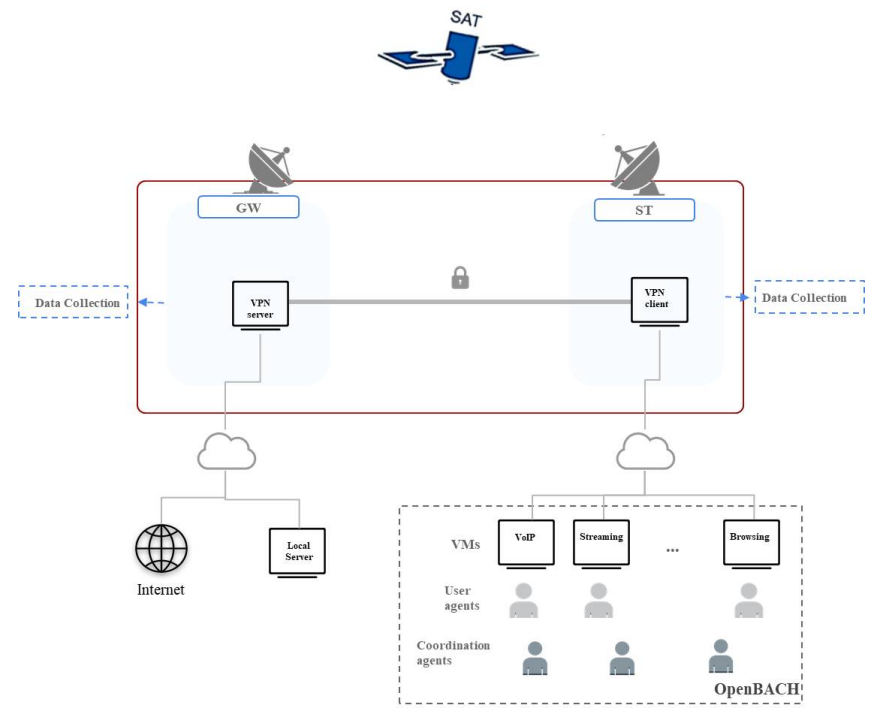

Fig. 6: Traffic emulation platform proposed in a Satellite Architecture.

The applications are launched in three main scenarios on the platform: i) Internet traffic without the tunnel ii) Unitary scenarios with the VPN: only one application at a time is launched, and ii) Multiple scenarios with the VPN: several applications are launched at the same time. Additionally, some network configurations were imposed on OpenSand. For each scenario, the data collection process was performed in the GW and ST, before and after the VPN. In this sense, all the possible transformations that the data perceived is recorded. The labeling process is performed per file and the application launched. However, for the VPN tunnel, a particular treatment was performed: for each packet getting into the tunnel, a flag was used to mark the application launched. Therefore,

\footnotetext{
${ }^{5}$ http://opensand.org/

${ }^{6}$ https://www.openbach.org/

7 https://www.seleniumhq.org/
} 
the multiplexed connections are correctly labeled. This dataset is still in development. In this particular work, we used only the data captured in the GW with the applications in Table 2 . These applications were launched differently to get a heterogeneous dataset; for instance, different codecs and websites were used for the VoIP and browsing applications, respectively. In Table 2, we show the flows captured per application and the number of packets with and without the VPN. It is important to mention that the duration varies from $5 \mathrm{~min}$ up to $15 \mathrm{~min}$. In addition to this, the experiments over the VPN were carried out using UDP as the transport protocol.

\begin{tabular}{|c|c|c|c|c|c|}
\cline { 3 - 6 } \multicolumn{2}{c}{} & \multicolumn{2}{c|}{ without VPN } & \multicolumn{2}{c|}{ with VPN } \\
\hline QoS class & Application & Flows & Packets & Packets: Unitary & Packets: Multiple \\
\hline \multirow{2}{*}{ VoIP } & facebook_voip & 302 & 227997 & 74904 & 522275 \\
& skype_voip & 565 & 315281 & 60764 & 673780 \\
& twinkle_voip & 69 & 141663 & 26144 & 276995 \\
\hline \multirow{2}{*}{ Video } & skype_video & 579 & 925391 & 318335 & 2235781 \\
& facebook_video & 357 & 558880 & 162822 & 1000071 \\
\hline Streaming & youtube_video_streaming & 760 & 158177 & 19619 & 486141 \\
\hline Browsing & web_browsing & 6852 & 749979 & 91705 & 1824852 \\
\hline Unknown & unknown & 58 & 2860 & 1080 & 2334 \\
\hline
\end{tabular}

Table 2: Class, packet and flow distribution of the SAT data in the GW.

\section{Experimental evaluation}

The training process was deployed by dividing the data as in Table 3. The complete data is used to build $D 1$, while for the rest of classifiers, the data is adapted according to their objectives. First of all, to create $C l 2$, we evaluate different time windows $\Delta t$ to find the most adequate. Afterward, we build the rest of the classifiers with different ML approaches. The best approaches are selected, and their average response time and accuracy are compared with nDPI.

\begin{tabular}{|c|c|c|c|}
\hline Classifier & \multicolumn{3}{|c|}{ All data } \\
\hline D1 & \multicolumn{2}{|c|}{ Without VPN } & With VPN \\
\hline Cl1 & Unencrypted & Encrypted & \\
\hline D2 & & unitary & multiple \\
\hline Cl2 & & unitary & \\
\hline MT & & & multiple \\
\hline
\end{tabular}

Table 3: Data settings for building the classifiers. 


\subsection{Classification system results}

Table 4 shows the results after evaluating different time windows for the unitary tunneled connections. The accuracy increase as $\Delta t$ does; therefore, we compare the average number of packets assessed for each application in Figure 7. We can notice that for $5 \mathrm{~ms}$ and $10 \mathrm{~ms}$, the amount of packets is very low. To avoid this, the new window will be adjustable in the sense that $\Delta t=10 \mathrm{~ms}$, but we wait until we have at least 20 packets to process.

\begin{tabular}{|c|c|c|}
\hline$\Delta t$ & Num. flow & Cl2 \\
\hline $5 \mathrm{~ms}$ & 167097 & 0.8982 \\
$10 \mathrm{~ms}$ & 120395 & 0.9647 \\
$100 \mathrm{~ms}$ & 26634 & 0.9673 \\
\hline
\end{tabular}

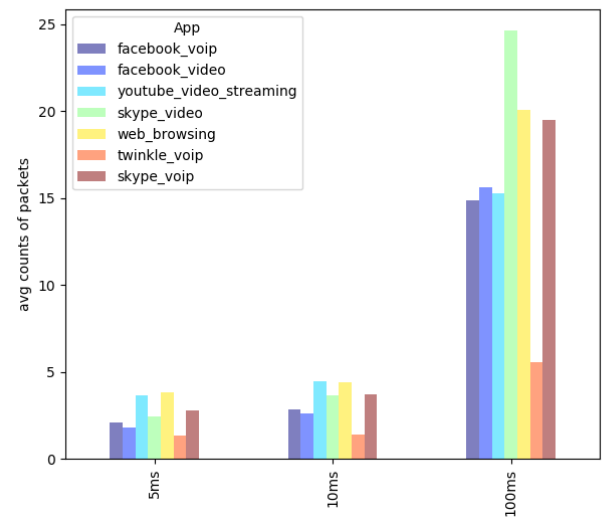

Table 4: Accuracy re- Fig. 7: Average counts of packets for sults for $C l 2$ varying $\Delta t$ each $\Delta t$

On the other hand, the results in Table 5 show a comparison between several classifiers: Decision Tree (DT), Random Forest (RF), K Nearest Neighbors (KNN), Ada Boost, Voting and Extra Trees (ETs). The best performance is standing up in bold. We picked DTs for the flow discrimination tasks, while RF for the traffic classification task.

\begin{tabular}{|c|c|c|c|c|c|c|}
\cline { 2 - 8 } \multicolumn{1}{c|}{} & DT & RF & KNN & AdaBoost & Voting & ETs \\
\hline D1 & $\mathbf{0 . 9 9 9 9}$ & 0.9999 & 0.9999 & 0.9999 & 0.9999 & 0.9999 \\
\hline Cl1 & 0.8876 & $\mathbf{0 . 9 1 8 6}$ & 0.8617 & 0.7986 & 0.8941 & 0.8938 \\
\hline D2 & 0.9588 & $\mathbf{0 . 9 6 4 6}$ & 0.9526 & 0.9584 & 0.9636 & 0.9638 \\
\hline Cl2 & 0.9321 & $\mathbf{0 . 9 4 0 1}$ & 0.9209 & 0.8333 & 0.9358 & 0.9304 \\
\hline
\end{tabular}

Table 5: Accuracy scores of several ML classifiers.

Following, the complete framework was implemented in C. The tree-based models are built in scikit-learn ${ }^{8}$ and parsed to $\mathrm{C}$ for faster Internet classifica-

\footnotetext{
${ }^{8}$ https://scikit-learn.org
} 
tions, inspired by the work in [4]. These tests were performed on a PC with an i7-6700HQ CPU and 32Gb RAM. The response time and accuracy are measured over the test set. We also evaluate nDPI for traffic classification.

In Table 6, we can notice that the $\mathrm{C}$ implemented models maintain their accuracy. In the unencrypted case, ML outperforms nDPI; while, for the encrypted example, nDPI is unable to detect the class of an unitary session as $C l 2$ does. Regarding the response time of the classifiers, in Table 7, we can notice that fast Internet classifications are possible. It is important to mention that the model response time differs for each entry depending on how deep they go into the tree's branches until a leaf is reached. In addition to this, the packet processing and flow metering response time varies from $5 \mathrm{~ms}$ to $15 \mathrm{~ms}$.

\begin{tabular}{|c|c|c|c|}
\cline { 3 - 3 } \multicolumn{2}{c|}{} & \multicolumn{2}{c}{ Acc } \\
\cline { 3 - 4 } \multicolumn{2}{c|}{} & ML & nDPI \\
\hline \multirow{2}{*}{ Unencrypted } & D1 & 0.9999 & 1 \\
& Cl1 & 0.9186 & 0.5830 \\
\hline \multirow{2}{*}{ Encrypted } & D2 & 0.9588 & $\mathrm{X}$ \\
& Cl2 & 0.9401 & $\mathrm{X}$ \\
\hline
\end{tabular}

Table 6: Accuracy (Acc) evaluating the test data

\begin{tabular}{|l|c|c|c|}
\multicolumn{2}{c}{} & \multicolumn{2}{c}{ Time $(\mu s)$} \\
\cline { 3 - 4 } \multicolumn{2}{c|}{} & ML & nDPI \\
\hline \multirow{2}{*}{ Unencrypted } & D1 & 2.867 & 1 \\
& Cl1 & 5 & 6.6460 \\
\hline \multirow{2}{*}{ Encrypted } & D2 & 2.717 & $\mathrm{X}$ \\
& Cl2 & 5 & $\mathrm{X}$ \\
\hline
\end{tabular}

Table 7: Average response time in $\mu s$

\subsection{About the multiplexed connections}

We were able to divide the multiplexed connections between unitary and nonunitary scenarios. We saw that classical ML approaches could classify the unitary scenarios. The scenario with multiple applications within a tunnel is a challenge in this field. To illustrate the problem, we take the unitary tunneled flows of Skype, YouTube, and Browsing; and its equivalent mixed tunneled flow. We represent them as a combination of types of packets (A: E from the source and 1:5 from the destination, using the packet lengths described in Table 1). We count the average number of packets for each combination within a time window of $100 \mathrm{~ms}$ and plot it into a heatmap. For instance, the flow "AAB1CAA" has $\mathrm{AA}: 2, \mathrm{AB}: 1, \mathrm{~B} 1: 1$ and CA:1. This representation is in Figure 8 . We can notice that the unitary tunneled connections have distinctively sequences of patterns that are merged in the mixed tunneled flow. It is important to say that the Skype pattern is maintained and might be identified. This illustration gives us an idea of how to decrypt the behavior within the tunneled connections by looking at the packet's patterns. However, the complexity grows when more than three applications are multiplexed in the tunnel. 


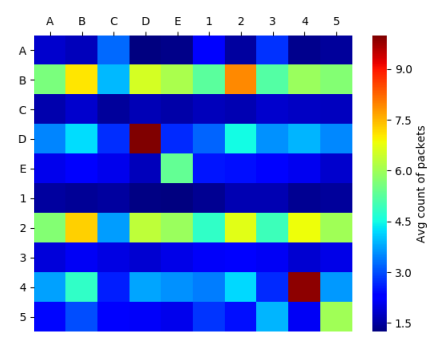

(a) Skype

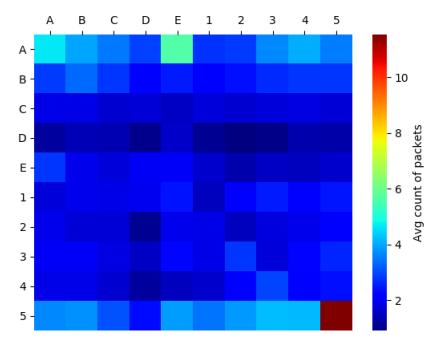

(c) Browsing

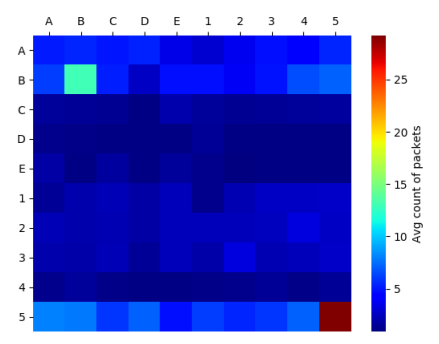

(b) YouTube

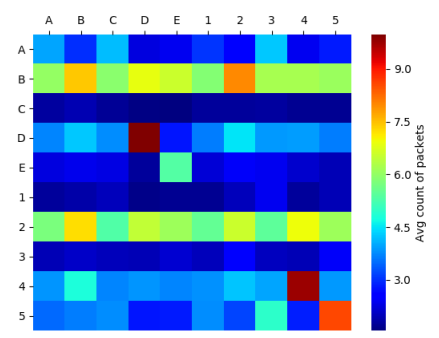

(d) Skype,YouTube and Browsing mixed

Fig. 8: Heatmap representation of the flows with $\Delta t=100 \mathrm{~ms}$.

\subsection{About the evolution of Internet traffic}

Most of the publicly available datasets do not comprise all the existing applications on the Internet; besides, the data collection process is tedious and expensive as remarked in [10]. One of the primary deficiencies of ML in this field is handling with the evolution of Internet traffic applications. If we consider some critical QoS classes such as YouTube, NetFlix, Skype, or Facebook video; as new incoming behavior, the classification accuracy might decrease considerably. Our architectural proposal comprises a component that should schedule retraining of the models when the network administrators demand it. But also, an automatic approach can be set to continuously modify the trees of the RFs in the Model repository component. Such an approach can be based on unsupervised methods for detecting Internet evolution.

\subsection{About the QoS management}

As we previously mentioned, it suffices to place the classification system over a network appliance that permits traffic monitoring. For instance, in the GW component, the classification output is forwarded to the PDP to perform the 
QoS management task. Depending on the classification output, QoS rules will be applied to trigger actions that will manage the Satellite resources. If a QoS rule is satisfied, the traffic will be shaped as follows:

- Aggregate flows: the QoS rule is applied over all the incoming packets sharing the same tuple $\left(I P_{s r c}, I P_{d s t}\right.$, port $_{s r c}$, port $_{d s t}$, proto $)$.

- Unitary tunneled flows: all the incoming packets of the unitary tunneled communications will be prioritized. However, this may be updated when the classification prediction of $D 2$ or $C l 2$ changes in $\Delta t$.

- Multiplexed tunneled flows: we can think about prioritizing the tunnel as the unitary case. Nevertheless, in parallel, other, less sensitive applications will also be benefited from this action. To avoid this, a classification per packet task should be designed.

In addition to this, we need to be sure that the QoS requirements are satisfied on time. For instance, according to [5], VoIP and Interactive video applications are susceptible to delivery delays, to be specific they can tolerate around $100 \mathrm{~ms}$; whereas, another critical class such as Video streaming no more than 10s. We notice that the classification task can be achieved in around $15 \mathrm{~ms}$, giving sufficient time to treat those sensitive classes.

\section{Conclusion}

This work presented an ML system that can be integrated into Internet traffic architectures, being the Satellite Architecture of our primary interest. The proposal can be comparable with an existing DPI solution, which offers a portable software solution for Internet traffic inspection. We tested our approach in the GW component, with data captured from an emulated Satellite platform. This approach outperformed in accuracy and time a well-known DPI solution. We displayed the needs of having components that can deal with the evolution of the Internet network and the multiplexed connections; these last aspects are in development. Future works also include implementing the approach in the emulated Satellite platform and tuning the framework proposed given different network conditions.

\section{Acknowledgment}

We want to thank the Centre National d'Études Spatiales (CNES), Toulouse, France for allowing us to use the SAT data, which is developed under the project R\&T CNES: Application du Machine Learning au Satcom.

\section{References}

1. Bertaux, L., Medjiah, S., Berthou, P., Abdellatif, S., Hakiri, A., Gelard, P., Planchou, F., Bruyere, M.: Software defined networking and virtualization for broadband satellite networks. IEEE Communications Magazine 53(3), 54-60 (March 2015) 
2. Deri, L., Martinelli, M., Bujlow, T., Cardigliano, A.: nDPI: Open-source highspeed deep packet inspection. In: 2014 International Wireless Communications and Mobile Computing Conference (IWCMC). pp. 617-622 (2014)

3. Ferrs, R., Koumaras, H., Sallent, O., Agapiou, G., Rasheed, T., Kourtis, M.A., Boustie, C., Glard, P., Ahmed, T.: Sdn/nfv-enabled satellite communications networks: Opportunities, scenarios and challenges. Physical Communication 18, 95 - 112 (2016), special Issue on Radio Access Network Architectures and Resource Management for $5 \mathrm{G}$

4. Garcia, J., Korhonen, T., Andersson, R., Vstlund, F.: Towards video flow classification at a million encrypted flows per second. In: 2018 IEEE 32nd International Conference on Advanced Information Networking and Applications (AINA). pp. 358-365 (May 2018)

5. ITU-T: End-user multimedia qos categories. Tech. rep., TELECOMMUNICATION STANDARDIZATION SECTOR OF ITU (2001)

6. Jin, Y., Duffield, N., Erman, J., Haffner, P., Sen, S., Zhang, Z.L.: A modular machine learning system for flow-level traffic classification in large networks. ACM Trans. Knowl. Discov. Data 6(1), 4:1-4:34 (Mar 2012)

7. Moore, B., Ellesson, E., Strassner, J., Westerinen, A.: Policy core information model - version 1 specification. https://tools. ietf .org/html/rfc3060, internet Engineering Task Force (IETF)

8. Ng, B., Hayes, M., Seah, W.K.G.: Developing a traffic classification platform for enterprise networks with sdn: Experiences amp;amp; lessons learned. In: 2015 IFIP Networking Conference (IFIP Networking). pp. 1-9 (May 2015)

9. Pacheco, F., Exposito, E., Aguilar, J., Gineste, M., Baudoin, C.: A novel statistical based feature extraction approach for the inner-class feature estimation using linear regression. In: 2018 International Joint Conference on Neural Networks (IJCNN). pp. 1-8 (July 2018)

10. Pacheco, F., Exposito, E., Gineste, M., Baudoin, C., Aguilar, J.: Towards the deployment of machine learning solutions in network traffic classification: A systematic survey. IEEE Communications Surveys Tutorials pp. 1-1 (2018)

11. Pacheco, F., Exposito, E., Gineste, M., Budoin, C.: An autonomic traffic analysis proposal using machine learning techniques. In: Proceedings of the 9th International Conference on Management of Digital EcoSystems. pp. 273-280. MEDES '17 (2017)

12. Pietrzyk, M., Costeux, J.L., Urvoy-Keller, G., En-Najjary, T.: Challenging statistical classification for operational usage: The adsl case. In: Proceedings of the 9th ACM SIGCOMM Conference on Internet Measurement. pp. 122-135. IMC '09 (2009)

13. Siller, M., Woods, J.C.: Qos arbitration for improving the qoe in multimedia transmission. In: 2003 International Conference on Visual Information Engineering VIE $2003(2003)$

14. Trestian, I., Ranjan, S., Kuzmanovic, A., Nucci, A.: Googling the internet: Profiling internet endpoints via the world wide web. IEEE/ACM Transactions on Networking 18(2), 666-679 (April 2010) 